MANCHESTER

1824

군

Economics

Discussion Paper Series

EDP-1114

A Genuine Foundation for Prospect Theory

Ulrich Schmidt

Horst Zank

May 2011

Economics

School of Social Sciences

The University of Manchester

Manchester M13 9PL 


\title{
A Genuine Foundation for Prospect Theory
}

\author{
by Ulrich Schmidt ${ }^{\mathrm{a}}$ and Horst Zank ${ }^{\mathrm{b}, 1}$ \\ a Department of Economics, Christian-Albrechts-Universität zu Kiel and Kiel Institute \\ for the World Economy, Germany. \\ ${ }^{b}$ Economics, School of Social Sciences, The University of Manchester, United Kingdom.
}

16 May 2011

\begin{abstract}
In previous models of (cumulative) prospect theory reference-dependence of preferences is imposed beforehand and the location of the reference point is exogenously determined. This note presents a principle that provides a critical test and a foundation for prospect theory preferences without assuming reference-dependent preferences. The latter is derived from behavior and a unique reference point arises endogenously.
\end{abstract}

Keywords: diminishing sensitivity, prospect theory, reference-dependence, reference point. Journal of Economic Literature Classification Numbers: D81.

\footnotetext{
${ }^{1}$ Correspondence: Horst Zank, Economics, The University of Manchester, Oxford Road, Manchester M13 9PL, United Kingdom; Telephone: ++44 161275 4872, Fax: ++44 161275 4812, E-mail: horst.zank@manchester.ac.uk
}

We thank Peter P Wakker for providing helpful remarks on an earlier draft of the paper. 


\section{Introduction}

Prospect theory (PT) is currently one of the most well known and very influential models of decision making under uncertainty (Wakker, 2010). It has been applied in various fields like finance, consumer choice, and political decision making. The central and most influential innovation of PT is reference-dependence. Reference-dependence means that people do not evaluate final outcomes but instead they base decisions on gains and losses relative to a reference point. As a result PT allows for the classification of risk attitudes into three major components: sign-dependent probability weighting, that is, the attitudes towards risk captured by the decision weights depends on the sign of outcomes; diminishing sensitivity, that is, people are more sensitive to changes near their reference points than to changes remote from it; and loss aversion, that is, a negative deviation from the reference point has a higher impact than a positive deviation of equal size.

Many empirically studies support reference-dependence by providing evidence on signdependence (Edwards 1953, 1954, Hogarth and Einhorn 1990, Tversky and Kahneman 1992, Abdellaoui 2000, Bleichrodt, Pinto, and Wakker 2001, Etchard-Vincent 2004, Payne 2005, Abdellaoui, Vossmann and Weber 2005, Abdellaoui, l'Haridon and Zank 2010), diminishing sensitivity (Kahneman and Tversky 1979, Tversky and Kahneman 1981, 1992, Hershey and Schoemaker 1985, Budescu and Weiss 1987, Camerer 1989, Currim and Sarin 1989, Fennema and van Assen 1999, Luce 2000, Abdellaoui 2000, Abdellaoui, Vossmann, and Weber 2005, Abdellaoui, Bleichrodt and Paraschiv 2007; studies in the field of neuro-economics include Dickhaut et al. 2003, de Martino et al. 2006), and loss aversion (Battalio, Kagel, and Jiranyakul 1990, Tversky and Kahneman 1992, Camerer 1998, Kahneman and Tversky 2000, Schmidt and Traub 2002, Camerer, Loewenstein and Rabin 
2004, Brooks and Zank 2005, Abdellaoui, Bleichrodt and Paraschiv 2007). Also for choice under certainty, reference dependence can be observed (Bateman et al. 1997) and serves as the key explanation for prominent phenomena like the disparity between willingness to pay and willingness to accept (Kahneman, Knetsch, and Thaler 1990, Bateman et al. 1997), the endowment effect (Thaler 1980, Loewenstein and Adler 1995), and the status quo bias (Samuelson and Zeckhauser, 1988).

We focus on reference-dependence under PT. Note that one of the major advantages that PT offers for empirical work is that it does not provide a clearly testable theory for the location of the reference point. Instead it allows the data to speak. This aspect of PT greatly improves its descriptive performance compared to other theories because it allows for sufficient degrees of freedom for the model to be fitted to a large body of data. From a theoretical point of view, not having a convincing theory for how the reference point emerges from choice behavior or testable preference conditions is a weakness of PT. Note that unlike original PT (Kahneman and Tversky 1979), modern variants like cumulative PT and the rank- and sign-dependent utility model (Luce 1991, Luce and Fishburn 1991, Tversky and Kahneman 1992, Wakker and Tversky 1993, Chateauneuf and Wakker 1999, Luce 2000, Zank 2001, Wakker and Zank 2002, Köbberling and Wakker 2003, Schmidt and Zank 2009) have been derived from behavioral foundations in terms of preference conditions.

The main motivation for this paper is the fact that currently PT lacks a testable axiomatization for the existence and location of the reference point. In our view this is an important and possibly fundamental issue for current axiomatizations of PT: all assume the existence of a preference relation defined on gains and losses relative to an exogenously fixed reference point and only then impose behavioral conditions on this 
(reference-dependent) preference relation in order to derive decision weights and utility. This means that reference-dependence is not derived form preference conditions but is assumed beforehand and as such could be regarded as an ad hoc assumption. As a consequence, PT can neither be tested nor applied to concrete choice problems without making prior assumptions about the location of the reference point. This is seen as a major shortcoming of PT; see, e.g., Fudenberg (2006, footnote 2 on page 696) and Pesendorfer (2006) for a discussion. The same criticism also applies to the reference-dependent model of Sugden (2003) and its generalization, third-generation PT (Schmidt, Starmer, and Sugden 2008). As far as we know, no theory exists which derives reference-dependence from preference conditions.

To date the only theories that provide endogenous reference points are those provided by Köszegi and Rabin (2006, 2007) and Köszegi (2010). These models are appealing for analyzing the implication of reference-dependent behavior in different contexts and for policy implications. These models are not based on normative or axiomatic motivations and difficult to test empirically, in particular as the reference points are expectations generated by a mechanism that cannot be observed (that is, the so-called "personal equilibrium" is a reference point that results from a mental game played in the decision makers brain). In this sense, these models come closer to decision models where the expectations are formed from the choices offered to the decision maker (e.g., regret or disappointment, Bell 1982, Loomes and Sugden 1982, Gul 1991). PT is different because it requires a reference point that is meaningful for all possible choice problems. Of course reference points can be manipulated through certain forms of framing (Kahneman and Tversky 1979), in certain contexts, or other mechanisms as, for example, used in game shows. But PT is set up to apply within a given frame, and, by taking the reference point as given, 
the existing preference foundation for PT are foundations within a given context.

We maintain a constant frame and extend existing axiomatizations of PT by providing preference conditions that dispense from the explicit coding of outcomes as gains and losses. What happens in the decision maker's mind can only be observed through certain choice behavior, and this aspect is key to our preference foundation. Because we work in the PT-framework we cannot claim that we provide a general theory of how reference points form. What we can claim, however, is that we provide a tool for testing whether preferences are displaying reference-dependence as predicted by PT. In particular, we provide a tool for verifying whether the risk attitudes captured by utility is exhibiting diminishing sensitivity.

We call the new approach endogenous prospect theory (EPT) because referencedependence is not assumed beforehand but derived from a preference foundation. In this sense we supplement existing PT foundations with a preference condition that allows the model to become testable independent of decision weighting. Our preferences conditions focus on utility. They imply the existence of a reference point and determine the location of the reference point endogenously. This requires a criterion for identifying the location of the reference point since reference-dependence becomes meaningless if behavior would not change at the reference point. As mentioned above, according to previous models of PT three criteria can be used to identify the reference point: sign-dependence, diminishing sensitivity or loss aversion. These criteria must be aligned with the theoretical approaches for deriving models for decision making under uncertainty, namely the "rich state space" approach or the "rich outcome set" approach.

A preference based tool to derive and test for reference-dependence via sign-dependence needs to be based on a preference axiomatization that focuses on the derivation of subjec- 
tive beliefs for the events of a sufficiently rich state space. This approach was pioneered by Savage (1954) and extended by Machina and Schmeidler (1992) deriving probabilistic sophistication. Approaches which allow for non-additive measures were provided by Gilboa (1987), Schmeidler (1989), and Abdellaoui and Wakker (2005). If probabilistic sophistication holds, the tools used in the models of Chateauneuf (1999), Abdellaoui (2002) and Zank (2010) could be supplemented with additional preference conditions that give sign-dependence, from which reference-dependence could be derived.

A more standard approach in economics is to provide axioms that focus on the derivation of continuous utility. Diminishing sensitivity and loss aversion can be aligned with this rich outcome set approach. We adopt the approach with continuous utility and focus on diminishing sensitivity. The alternative approach of using loss aversion to identify the reference point is left for future research. Loss aversion has been used, e.g., by Abeler, et al. (2011) to test the model of Köszegi and Rabin 2006, 2007, and Köszegi 2010, and we think that testing for reference-dependence via loss aversion is a promising approach too. But its implementation is not straightforward. For example, loss aversion implies the existence of a kink at the reference point. This kink needs to be distinguished from possible genuine kinks of the utility function under PT. This would require additional information about the curvature of utility as with diminishing sensitivity, the approach that we adopt. Further, there exist different definition of loss aversion in the literature (for an overview see Abdellaoui, Bleichrodt, and Paraschiv 2007), and behavioral foundations of these definitions of loss aversion are often missing or they have model-dependent implications (Schmidt and Zank 2005, 2008). Some definitions of loss aversion based on utility require the identification of utility for gains and for losses first, and only then one can compare absolute or marginal changes in utility for gains and losses of similar size. 
It is, therefore, not immediately clear how a principle that rests on loss aversion can be developed without in-advance knowledge of the reference point. Nonetheless, loss aversion is an important feature of PT and is incorporated in our model.

The next section introduces our framework of decision making under uncertainty and some basic concepts. Section 3 contains our behavioral conditions and the main result: By imposing our central axiom -termed consistent diminishing sensitivity- referencedependence arises endogenously in our model and the reference point is located at the position at which sensitivity towards changes in outcomes is maximal. Concluding remarks are presented in Section 4.

\section{Notation and Basic Concepts}

We analyze decision problems under uncertainty and consider a finite set $S$ of states of nature. ${ }^{2}$ That is, $S=\left\{s_{1}, \ldots, s_{n}\right\}$ for a natural number $n \geq 3$, and $\mathcal{A}=2^{S}$ is the algebra of subsets of $S$. Elements of $\mathcal{A}$ are called events. An act $f$ assigns to each state a real valued outcome. The set of acts $\mathcal{F}$ can be identified with the Cartesian product space $\mathbb{R}^{\ltimes}$, and hence, we write $f=\left(f_{1}, \ldots, f_{n}\right)$, where $f_{i}$ is short for $f\left(s_{i}\right)$. An act $f$ is rank-ordered if its outcomes are ordered from best to worst: $f_{1} \geq \cdots \geq f_{n}$.

For each act $f$ there exists a permutation $\rho$ of $\{1, \ldots, n\}$ such that $f_{\rho(1)} \geq \cdots \geq f_{\rho(n)}$, i.e. such that the outcomes are rank-ordered with respect to $\rho$. For each permutation $\rho$ of $\{1, \ldots, n\}$ the set $\mathbb{R}_{\rho}^{\times}$consists of those acts which are rank-ordered with respect to $\rho$. Acts that can be rank-ordered with respect to the same permutation are called comonotonic.

\footnotetext{
${ }^{2}$ Our results can be extended to infinite state spaces by using tools presented in Wakker (1993). Identical results for the case of decision under risk, that is, under probabilistic sophistication or when (objective) probabilities are given, can be derived by applying the procedure of Köbberling and Wakker (2003, Section 5.3).
} 
We use the notation $f_{E} g$ for an act that agrees with the act $f$ on event $E$ (i.e., $f(s)=f_{E} g(s)$ for all $\left.s \in E\right)$ and agrees with the act $g$ on the complement of $E, E^{c}$. Also, we use $h_{i} f$ instead of $h_{\left\{s_{i}\right\}} f$ for any state $s_{i} \in S$. Sometimes we identify constant acts with the corresponding outcome. We may thus write $f_{E} x$ for an act agreeing with $f$ on $E$ and giving outcome $x$ for states $s \in E^{c}$.

We consider a preference relation $\succcurlyeq$ on the set of acts. As usually, $f \succcurlyeq g$ means that the act $f$ is weakly preferred to the act $g$. The symbols $\succ$ and $\sim$ denote strict preference and indifference, respectively. The preference relation $\succcurlyeq$ is a weak order if it is complete $(f \succcurlyeq g$ or $g \succcurlyeq f$ for any acts $f, g)$ and transitive. A functional $V: \mathcal{F} \rightarrow \mathbb{R}$ represents the preference relation $\succcurlyeq$ if for all $f, g \in \mathcal{F}$ we have $f \succcurlyeq g \Leftrightarrow V(f) \geqslant V(g)$.

An example of a representing functional is Choquet expected utility (CEU) introduced by Schmeidler (1989) and Gilboa (1987). It extends the classical subjective expected utility of Savage (1954) by introducing a non-additive measure for events: a capacity $v$ satisfies $v(S)=1, v(\emptyset)=0$, and $v(A) \geq v(B)$ if $A \supseteq B$ and $A, B \in \mathcal{A}$. A capacity $v$ is strictly monotonic if $v(A)>v(B)$ for $A \supsetneqq B$ and $A, B \in \mathcal{A}$.

Choquet expected utility holds if the preference relation can be represented by the functional

$$
C E U(f)=\sum_{i=1}^{n} U\left(f_{i}\right) \pi_{i} \quad \text { with } \quad \pi_{i}=v\left(\left\{s_{\rho(1)}, \ldots, s_{\rho(i)}\right\}\right)-v\left(\left\{s_{\rho(1)}, \ldots, s_{\rho(i-1)}\right\}\right)
$$

The strictly increasing and continuous utility, $U$, is cardinal (i.e., it can be replaced by a positive linear transformation of $U$ ) and the capacity, $v$, is unique. In terms of behavioral conditions, CEU can be derived by restricting Savage (1954)'s sure-thing principle to acts which are pair-wise comonotonic, and further by requiring a consistent ordering of utility differences across states (see Köbberling and Wakker 2003). 
Prospect theory generalizes CEU by introducing a reference point $r$, which may impact on utility and capacity. In all axiomatic work we are aware of, the existence and location of this reference point is given from outset. Formally, previous models considered a preference relation $\succcurlyeq_{r}$ on acts with outcomes being deviations from $r$, i.e. for the act $f$ the outcome $f_{i}$ is interpreted as gain (loss) if it is better (worse) than $r$.

Prospect Theory (PT) holds if the representing functional for $\succcurlyeq_{r}$ has the form

$$
\begin{aligned}
P T(f) & =\sum_{i=1}^{n} U\left(f_{i}\right) \pi_{i}, \\
\text { with } \pi_{i}= & \left\{\begin{array}{l}
v^{+}\left(\left\{s_{\rho(1)}, \ldots, s_{\rho(i)}\right\}\right)-v^{+}\left(\left\{s_{\rho(1)}, \ldots, s_{\rho(i-1)}\right\}\right) \text { if } f_{i} \geq r \\
v^{-}\left(\left\{s_{\rho(i)}, \ldots, s_{\rho(n)}\right\}\right)-v^{-}\left(\left\{s_{\rho(i+1)}, \ldots, s_{\rho(n)}\right\}\right) \text { if } f_{i} \leq r .
\end{array}\right.
\end{aligned}
$$

The two (possibly different) capacities $v^{+}$and $v^{-}$are uniquely determined and the utility is a ratio scale (i.e., unique up to multiplication by a positive constant) as it is fixed at the reference point, i.e., $U(r)=0$.

In the next section we impose preference conditions for a general preference over acts and derive reference-dependence and PT from these conditions.

\section{A New Foundation for Prospect Theory}

The primitives in this section is a preference $\succcurlyeq$ over acts in $\mathcal{F}$. We do not impose a reference point, hence outcomes are not subject to an interpretation of gains or losses. Information of how outcomes are perceived follows from behavior and is captured in our main preference condition.

Let us first recall some standard properties for the preference $\succcurlyeq$, before we introduce the main preference condition that allows identifying the reference point. The preference 
relation $\succcurlyeq$ on $\mathcal{F}$ satisfies monotonicity if $f \succ g$ whenever $f_{i} \geq g_{i}$ for all states $s_{i}$ with a strict inequality for at least one state. By employing this condition we ensure that the capacities, derived later, are strictly monotone because monotonicity excludes null states, that is, states where the preference is independent of the magnitude of outcomes. Formally, a state $s_{i}$ is null if $x_{i} f \sim y_{i} f$ for all acts $f$ and all outcomes $x, y$.

The continuity condition defined here is continuity with respect to the Euclidean topology on $\mathbb{R}^{\ltimes}: \succcurlyeq$ satisfies continuity if for any act $f$ the sets $\{g \in \mathcal{F} \mid g \succcurlyeq f\}$ and $\{g \in \mathcal{F} \mid g \preccurlyeq f\}$ are closed subsets of $\mathbb{R}^{\ltimes}$.

In what follows we use several indifferences of the form $x_{i} f \sim y_{i} g$ with the assumption that all acts involved in such indifferences are rank-ordered with respect to the same permutation $\rho$. This indifference says that the difference in preference between the acts $f$ and $g$ outside state $s_{i}$ is off-set by receiving $x$ and $y$, for the respective acts, if state $s_{i}$ occurs. A equalizing tradeoff $x$ for $y$ is obtained given the acts $f, g$ and the state $s_{i}$. Whether $x$ or $y$ are gains or losses is not known at this stage but can be revealed through consistent behavior.

We can now introduce the main condition in the paper. Consistent diminishing sensitivity holds if for each outcome $x$ one of the following holds:

(I) for any outcomes $w, z, y$ larger than $x$

$$
\begin{aligned}
\text { if } x_{j} f & \sim y_{j} g \text { and } z_{j} f \sim w_{j} g, \\
\text { then } w-z & >y-x \\
\text { and further } x_{i} f^{\prime} & \sim y_{i} g^{\prime} \text { implies } z_{i} f^{\prime} \sim w_{i} g^{\prime}
\end{aligned}
$$

whenever all acts involved are rank-ordered with respect to the same permutation $\rho$ 
(II) for any outcomes $w, z, y$ smaller than $x$

$$
\begin{aligned}
\text { if } x_{j} f & \sim y_{j} g \text { and } z_{j} f \sim w_{j} g, \\
\text { then } z-w & >x-y \\
\text { and further } x_{i} f^{\prime} & \sim y_{i} g^{\prime} \text { implies } z_{i} f^{\prime} \sim w_{i} g^{\prime}
\end{aligned}
$$

whenever all acts involved are rank-ordered with respect to the same permutation $\rho$.

In the presence of weak order, monotonicity and continuity, one can always find acts $f$ and $g$ and distinct outcomes $w, z, y, x$ such that the indifferences $x_{j} f \sim y_{j} g$ and $z_{j} f \sim w_{j} g$ in statements (I) or (II) hold. One observes that the second indifference is obtained from the first by replacing $x$ and $y$ with $z$ and $w$, respectively. Consistent diminishing sensitivity puts constraints on the relationship between $(y-x)$ and $(w-z)$ as explained next.

Suppose that $x$ is such that the property (I) of consistent diminishing sensitivity holds. Further, assume that increasing $x$ in state $s_{j}$ of act $f$ to $z$ is as good as increasing $y$ in state $s_{j}$ of act $g$ to a larger outcome $w$. Then, consistent diminishing sensitivity requires two aspects. First, a larger increment than $y-x$ is needed to obtain the second indifference and, hence, $w-z>y-x$. Second, this larger increment is required to be independent of the (pair of) acts $f$ and $g$ and the state $s_{j}$, so that the strict inequality is consistent across states. Such a finding is in agreement with risk aversion in the sense of diminishing marginal utility for increments in outcomes. For example, under PT this is supposed to hold only for gains, where utility is concave, and it then implies that $x$ is a gain too.

Suppose, however, that $x$ is such that the property (II) of consistent diminishing sensitivity holds. Then those indifferences say that decreasing $x$ in state $s_{j}$ of act $f$ to $z$ is as bad as decreasing outcome $y$ in state $s_{j}$ of act $g$ to a smaller $w$. The property now 
requires that a larger decrement than $x-y$ is needed to obtain the second indifference and, hence, $z-w>x-y$. Similarly to the previous case, this larger decrement is required to be independent of the acts $f$ and $g$ and the state $s_{j}$. This latter finding is in agreement with risk seeking in the sense of diminishing marginal utility for decrements in outcomes, and under PT is supposed to hold for losses, thereby implying that $x$ must be a loss.

Recall that consistent diminishing sensitivity does not require a distinction of outcomes into gains and losses. It only says that for each outcome $x$ one of the statements (I) or (II) above must hold. It may, therefore, occur that for all outcomes only the part (I) holds. Or, it may be the case that for all outcomes only part (II) holds. It is worth noting at this stage that, in the presence of the other standard properties, if there exists some $x$ for which constraint (I) is satisfied, then (I) must be satisfied for all $x^{\prime}>x$; and if there exists some $x$ for which the second constraint (II) is satisfied, then (II) is satisfied for all $x^{\prime}<x$. It, therefore, follows that if there exists an outcome $x^{+}$for which (I) holds and an outcome $x^{-}$for which (II) holds, then there exists a unique outcome $r$ for which both (I) and (II) must hold. As behavior changes radically at this outcome, behavior reveals that $r$ must be the reference point for the preference $\succcurlyeq$, and that $\succcurlyeq$ exhibits referencedependence. Under PT this implies the well-documented S-shape of utility: concave for outcomes above $r$ and convex for outcomes below $r$.

The following calculus illustrates how CEU and PT imply consistent diminishing sensitivity. We distinguish 3 cases: (A) First, suppose that CEU holds and that utility is strictly concave. Then substitution of CEU for the indifferences $x_{j} f \sim y_{j} g$ and $z_{j} f \sim w_{j} g$ and subtracting the first resulting equality from the second implies

$$
U(y)-U(x)=U(w)-U(z)
$$


The additional requirement of strict concavity for utility implies that $w-z>y-x$ must hold. Recall that such preferences can be interpreted as PT preferences with the reference point being at minus infinity (that is, all outcomes are gains). Further, it must hold that $x_{i} f^{\prime} \sim y_{i} g^{\prime}$ implies $z_{i} f^{\prime} \sim w_{i} g^{\prime}$ for otherwise the above equality is violated. This implies that for each outcome $x$ statement (I) of consistent diminishing sensitivity holds.

In the second case (B) we assume that CEU holds with a strictly convex utility. Such preferences can then be interpreted as PT preferences with the reference point being at infinity (that is, all outcomes are seen as being losses). Similarly to case (A) it now follows that for each outcome $x$ statement (II) of consistent diminishing sensitivity holds.

For the third case $(\mathrm{C})$ suppose that there exists an outcome $r$ such that preferences are represented by PT with $U$ strictly concave (convex) for $f(s) \geq r(f(s) \leq r)$. Then, substitution of PT for the indifferences $x_{j} f \sim y_{j} g$ and $z_{j} f \sim w_{j} g$ and subtracting the first resulting equality from the second implies

$$
U(y)-U(x)=U(w)-U(z)
$$

whenever $w, z, y>x \geq r$ and the strict concavity of $U$ implies $w-z>y-x$. Further, $x_{i} f^{\prime} \sim y_{i} g^{\prime}$ implies $z_{i} f^{\prime} \sim w_{i} g^{\prime}$, for otherwise the above equality is violated.

We also have

$$
U(y)-U(x)=U(w)-U(z)
$$

whenever $w, z, y<x \leq r$ and the strict convexity of $U$ implies $z-w>x-y$. Further, $x_{i} f^{\prime} \sim y_{i} g^{\prime}$ implies $z_{i} f^{\prime} \sim w_{i} g^{\prime}$, for otherwise the above equality is violated. We conclude that in this case both statements (I) and (II) of consistent diminishing sensitivity hold at $r$.

The representing functional that agrees with either $(\mathrm{A})$ or $(\mathrm{B})$ or $(\mathrm{C})$ is called endoge- 
nous prospect theory (EPT). Note that consistent diminishing sensitivity is a necessary condition for EPT. The following theorem shows that, in the presence of the other standard preference conditions, consistent diminishing sensitivity is also sufficient for EPT. This is the main result of the paper:

THEOREM 1 Suppose that $\succcurlyeq$ is a preference relation on $\mathbb{R}^{\ltimes}, \ltimes \geq \nVdash$. Then the following two statements are equivalent:

(i) EPT holds with strictly monotone capacities.

(ii) The preference relation $\succcurlyeq$ is a monotonic, continuous weak order satisfying consistent diminishing sensitivity.

Utility is a ratio scale and the capacities are unique. If the reference point $r$ is finite it is uniquely determined.

The proof of Theorem 1 is presented in the Appendix.

\section{Conclusion}

From a theoretical point of view, the mathematical tools used in our theory build on existing tools that were used to derive PT with exogenous reference points. The advances proposed in this paper are conceptually important. For PT to become a valuable tool for economic analyses the model needs a theoretical foundation of how to detect the reference point from preferences. This is a shortcoming in earlier derivations of PT, which has often been criticized, and our note proposes a solution that overcomes this hurdle. We think that this makes PT more sound as a theory and more acceptable. At the same time, this note clarifies on a fundamental aspect on PT: like the classical subjective expected utility and other models of choice under uncertainty and ambiguity, PT belongs to the same family of models which are founded on common assumptions about preferences over uncertain 
acts, as there is no need to take reference-dependent preferences as primitive - the latter follows from the observable or revealed (risk) behavior.

\section{Appendix: Proof}

To prove Theorem 1 we remark that deriving statement (ii) from statement (i) is standard in conjunction with the comments preceding Theorem 1 regarding consistent diminishing sensitivity. Next we assume statement (ii) and derive statement (i). We distinguish three cases:

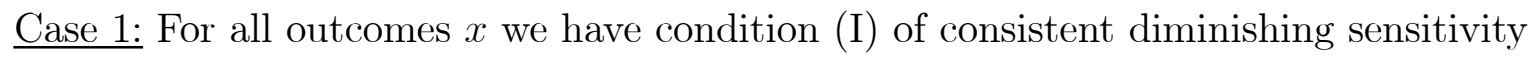
satisfied. In this case the comonotonic tradeoff consistency of Köbberling and Wakker (2003) holds and it follows from their Theorem 8 that CEU holds (with uniqueness results as noted in Observation 9 (c) of Köbberling and Wakker). Further, locally, we can always find indifferences $x_{j} f \sim y_{j} g$ and $z_{j} f \sim w_{j} g$ for acts $f, g$ a state $j$ and outcomes $w, z, y>x$. Substitution of CEU and subtraction of the first resulting equality from the second implies

$$
U(y)-U(x)=U(w)-U(z)
$$

Constant diminishing sensitivity demands $w-z>y-x$ in this case. Because this implication must hold for any outcome $x$ (and corresponding outcomes $w, z, y>x$ ), it follows, first locally and then globally, that the utility function must be concave.

Case 2: For all $x$ we have condition (II) of consistent diminishing sensitivity satisfied. Similar to the previous case, the results of Köbberling and Wakker (2003) hold and we obtain CEU. Further, consistent diminishing sensitivity implies, first locally and then globally, that the utility function is convex. Uniqueness results apply as noted in Observation 9 (c) of Köbberling and Wakker (2003). 
Case 3: There exist an outcome $x^{+}$for which condition (I) of constant diminishing sensitivity holds and an outcome $x^{-}$for which condition (II) of consistent diminishing sensitivity holds. It then follows that there exists a unique outcome $r$ for which both (I) and (II) must hold, which is the reference point for the preference $\succcurlyeq$. In this case consistent diminishing sensitivity implies the sign-comonotonic tradeoff consistency of Köbberling and Wakker (2003), and from their Theorem 12 we obtain that PT holds. By Proposition 8.2 in Wakker and Tversky (1993) the gain-loss consistency requirement can be dropped from statement (ii) in Theorem 12 in Köbberling and Wakker's (2003) when the number of states of nature exceeds 2 , which is the case here. Similar to cases 1 and 2 above we derive strict concavity of utility for outcomes above $r$ and strict convexity for utility for outcomes below $r$, first locally and then globally. Uniqueness results follow from Observation 13 in Köbberling and Wakker (2003).

Together cases 1-3 cover all possibilities and thus statement (i) follows in conjunction with the uniqueness results. This completes the proof of the theorem.

\section{References}

Abdellaoui, Mohammed. 2000. "Parameter-free Elicitation of Utility and Probability Weighting Functions." Management Science 46, 1497-1512.

Abdellaoui, Mohammed. 2002. "A Genuine Rank-Dependent Generalization of the von Neumann-Morgenstern Expected Utility Theorem," Econometrica 70, 717-736.

Abdellaoui, Mohammed, Han Bleichrodt, and Corina Paraschiv. 2007. "Measuring Loss Aversion under Prospect Theory: A Parameter-free Approach." Management 
Science 53, 1659-1674.

Abdellaoui, Mohammed, Olivier l'Haridon and Horst Zank. 2010. "Separating Curvature and Elevation: A Parametric Probability Weighting Function," Journal of Risk and Uncertainty 41, 39-65.

Abdellaoui, Mohammed, Frank Vossmann, and Martin Weber. 2005. "Choice-based Elicitation and Decomposition of Decision Weights for Gains and Losses under Uncertainty." Management Science 51, 1384-1399.

Abdellaoui, Mohammed and Peter P. Wakker. 2005. "The Likelihood Method for Decision under Uncertainty," Theory and Decision 58, 3-76.

Abeler, Johannes, Armin Falk, Lorenz Goette, and David Huffman. 2011. "Reference Points and Effort Provision," American Economic Review 101, 470-492.

Bateman, Ian J., Alistair Munro, Bruce Rhodes, Chris Starmer and Robert Sugden. 1997. "A Test of the Theory of Reference-Dependent Preferences," Quarterly Journal of Economics 112, 647-661.

Battalio, Raymond C., John H. Kagel and Komain Jiranyakul. 1990. "Testing Between Alternative Models of Choice Under Uncertainty: Some Initial Results," Journal of Risk and Uncertainty 3, 25-50.

Bell, David E. 1982. "Regret in Decision Making under Uncertainty," Operations Research 30, 961-981.

Bleichrodt, Han, Jose Luis Pinto and Peter P. Wakker. 2001. "Making Descriptive Use of Prospect Theory to Improve the Prescriptive Use of Expected Utility," Management 
Science 47, 1498-1514.

Brooks, Peter and Horst Zank. 2005. "Loss Averse Behavior," Journal of Risk and Uncertainty 31, 301-325.

Budescu, David V. and Wendy Weiss. 1987. "Reflection of Transitive and Intransitive Preferences: A Test of Prospect Theory." Organizational Behavior and Human Decision Processes 39, 184-202.

Camerer, Colin F. 1989. "An experimental test of several generalized utility theories," Journal of Risk and Uncertainty 2, 61-104.

Camerer, Colin F. (1998), "Bounded Rationality in Individual Decision Making," Experimental Economics 1, 163-183.

Camerer, Colin F., George Loewenstein and Matthew Rabin. 2004. Advances in Behavioral Economics, Princeton University Press, Princeton, NJ.

Chateauneuf, Alain. 1999. "Comonotonicity Axioms and Rank-Dependent Expected Utility for Arbitrary Consequences," Journal of Mathematical Economics 32, 2145.

Chateauneuf, Alain and Peter P. Wakker. 1999. "An Axiomatization of Cumulative Prospect Theory for Decision under Risk." Journal of Risk and Uncertainty 18, 137-145.

Currim, Imran S. and Rakesh K. Sarin. 1989. "Prospect versus Utility." Management Science 35, 22-41. 
de Martino, Benedetto, Dharshan Kumaran, Ben Seymour, and Raymond J. Dolan. 2006. "Frames, Biases, and Rational Decision-making in the Human Brain." Science 313, August 4, 684-687.

Dickhaut, John W., Kevin McCabe, Jennifer C. Nagode, Aldo Rustichini, Kip Smith, and José V. Pardo. 2003. "The Impact of the Certainty Context on the Process of Choice." Proceedings of the National Academy of Sciences 100, 3536-3541.

Edwards, Ward. 1953. "Probability-Preferences in Gambling," Americal Journal of Psychology 66, 349-364.

Edwards, Ward. 1954. "Probability-Preferences Among Bets With Differing Expected Values," Americal Journal of Psychology 67, 56-67.

Etchart-Vincent, Nathalie. 2004. "Is Probability Weighting Sensitive to the Magnitude of Consequences? An Experimental Investigation on Losses," Journal of Risk and Uncertainty 28, 217-235.

Fennema, Hein and Marcel van Assen. 1999. "Measuring the Utility for Losses by Means of the Tradeoff Method," Journal of Risk and Uncertainty 17, 277-295.

Fudenberg, Drew. 2006. "Advancing beyond Advances in Behavioral Economics." Journal of Economic Literature 44, 694-711.

Gilboa, Itzhak. 1987. "Expected Etility with Purely Subjective Non-additive Probabilities." Journal of Mathematical Economics 16, 65-88.

Gul, Faruk. 1991. "A Theory of Disappointment Aversion," Econometrica 59, 667-686. 
Hershey, John C. and Paul J. H. Schoemaker. 1985. "Probability versus Certainty Equivalence Methods in Utility Measurement: Are they Equivalent?" Management Science 31, 1213-1231.

Hogarth, Robin M. and Hillel J. Einhorn. 1990. "Venture Theory: A Model of Decision Weights," Management Science 36, 780-803.

Kahneman, Daniel, Jack L. Knetsch and Richard H. Thaler. 1990. "Experimental Tests of the Endowment Effect and the Coase Theorem," Journal of Political Economy $98,1325-1348$.

Kahneman, Daniel and Amos Tversky. 1979. "Prospect Theory: An Analysis of Decision under Risk," Econometrica 47, 263-291.

Kahneman, Daniel and Amos Tversky. 2000. Choices, Values, and Frames. Cambridge University Press, New York.

Köbberling, Veronika and Peter P. Wakker. 2003. "Preference Foundations for Nonexpected Utility: A Generalized and Simplified Technique." Mathematics of Operations Research 28, 395-423.

Köszegi, Botond and Matthew Rabin. 2006. "A Model of Reference-Dependent Preferences." Quarterly Journal of Economics 121, 1133-1165.

Köszegi, Botond and Matthew Rabin. 2007. "Reference-dependent Risk Attitudes." American Economic Review 97, 1047-1073.

Köszegi, Botond. 2010. "Utility from Anticipation and Personal Equilibrium," Economic Theory 44, 415-444. 
Loewenstein, George F. and Daniel Adler 1995. "A Bias in the Prediction of Tastes," Economic Journal 105, 929-937.

Loomes, Graham \& Robert Sugden. 1982. "Regret Theory: An Alternative Theory of Rational Choice under Uncertainty," Economic Journal 92, 805-824.

Luce, R. Duncan. 1991. "Rank-and-sign Dependent Linear Utility Models for Binary Gambles." Journal of Economic Theory 53, 75-100.

Luce, R. Duncan. 2000. Utility of Gains and Losses: Measurement-Theoretical and Experimental Approaches. New Jersey: Lawrence Erlbaum Associates.

Luce, R. Duncan and Peter C. Fishburn. 1991. "Rank- and Sign-dependent Linear Utility Models for Finite First-order Gambles." Journal of Risk and Uncertainty 4, $29-59$.

Machina, Mark J. and David Schmeidler. 1992. "A More Robust Definition of Subjective Probability," Econometrica 60, 745-780.

Payne, John W. 2005. "It is Whether You Win or Lose: The Importance of the Overall Probabilities of Winning or Losing in Risky Choice," Journal of Risk and Uncertainty 30, 5-19.

Pesendorfer, Wolfgang. 2006. "Behavioral Economics Comes of Age." Journal of Economic Literature 44, 712-721.

Samuelson, William F. and Richard J. Zeckhauser 1988. "Status Quo Bias in Decision Making," Journal of Risk and Uncertainty 1, 7-59.

Savage, Leonard J. 1954. The Foundations of Statistics. New York: Wiley. 
Schmeidler, David. 1989. "Subjective Probability and Expected Utility without Additivity." Econometrica 57, 571-587.

Schmidt, Ulrich. 2003. "Reference-dependence in Cumulative Prospect Theory." Journal of Mathematical Psychology 47, 122-131.

Schmidt, Ulrich, Chris Starmer, and Robert Sugden. 2008. "Third-Generation Prospect Theory," Journal of Risk and Uncertainty 36, 203-223.

Schmidt, Ulrich and Stefan Traub. 2002. "An Experimental Test of Loss Aversion," Journal of Risk and Uncertainty 25, 233-249.

Schmidt, Ulrich and Horst Zank. 2005. "What is Loss Aversion?" Journal of Risk and Uncertainty 30, 157-167.

Schmidt, Ulrich and Horst Zank. 2008. "Risk Aversion in Cumulative Prospect Theory." Management Science 54, 208-216.

Schmidt, Ulrich and Horst Zank. 2009. "A Simple Model of Cumulative Prospect Theory." Journal of Mathematical Economics 45, 308-319.

Starmer, Chris and Robert Sugden. 1989. "Violations of the Independence Axiom in Common Ratio Problems: An Experimental Test of Some Competing Hypotheses." Annals of Operations Research 19, 79-102.

Sugden, Robert. 2003. "Reference-dependent Subjective Expected Utility," Journal of Economic Theory 111, 172-191.

Thaler, Richard H. 1980. "Toward a Positive Theory of Consumer Choice," Journal of Economic Behavior and Organization 1, 39-60. 
Tversky, Amos and Daniel Kahneman. 1991. "Loss Aversion in Riskless Choice: A Reference-dependent Model." Quarterly Journal of Economics 56, 1039-1061.

Tversky, Amos and Daniel Kahneman. 1992. "Advances in Prospect Theory: Cumulative Representation of Uncertainty." Journal of Risk and Uncertainty 5, 297-323.

Wakker, Peter P. 1993. "Unbounded Utility for Savage's "Foundation of Statistics," and Other Models." Mathematics of Operations Research 18, 446-485.

Wakker, Peter P. 2010. Prospect Theory: For Risk and Ambiguity. Cambridge University Press, Cambridge, UK

Wakker, Peter P. and Amos Tversky. 1993. "An Axiomatization of Cumulative Prospect Theory." Journal of Risk and Uncertainty 7, 147-176.

Wakker, Peter P. and Horst Zank. 2002. "A Simple Preference-foundation of Cumulative Prospect Theory with Power Utility." European Economic Review 46, 1253-1271.

Zank, Horst. 2001. "Cumulative Prospect Theory for Parametric and Multiattribute Utilities." Mathematics of Operations Research 26, 67-81.

Zank, Horst. 2010. "Consistent Probability Attitudes," Economic Theory 44, 167-185. 